\title{
Clinical Significance of Cyclophosphamide-induced Cardiotoxicity
}

Key words: cardiotoxicity, hemorrhagic myocarditis, chemotherapeutic agents, cyclophosphamide

Cardiotoxicity can be induced by a number of chemotherapeutic agents and particularly by anthracyclines, such as doxorubicin. Doxorubicin-induced cardiotoxicity is recognized to follow a chronic clinical course that depends on cumulative dose. On the other hand, cardiotoxicity due to cyclophosphamide (CY) was initially described as a complication of bone marrow transplantation by Santos et al (1) in 1972. In protocols of bone marrow transplantation and peripheral blood stem cell transplantation (PBSCT), high-dose chemotherapy, including CY, is largely employed. High doses of CY, however, rarely cause serious cardiotoxicity and cardiac death. In contrast to doxorubicin-induced cardiotoxicity, high-dose $\mathrm{CY}$ causes acute cardiac dysfunction that is independent of cumulative dose. Acute heart failure secondary to cardiotoxicity has been reported about 1 week after CY administration, and the incidence rate is about $20 \%$ and mortality about $8 \%$ after bone marrow transplantation $(2,3)$. Although the mortality is high, survivors may show no residual cardiac abnormality.

The pathogenesis of CY-induced cardiotoxicity is still poorly understood, however, it is thought to involve direct endothelial damage, leading in turn to leakage of plasma proteins and erythrocytes. The histological findings indicate acute pericarditis and hemorrhagic myocarditis with fibrinplatelet microthrombi in capillaries and fibrin strands in the interstitium on ultrastructural examination (4). Wall thickening due to interstitial edema and hemorrhage may reduce left ventricular (LV) diastolic compliance as LV diastolic dysfunction and present as restrictive cardiomyopathy. In patients undergoing high-dose CY therapy who show evidence of clinical and radiological cardiac failure, hemorrhagic myocarditis should be suspected and carefully considered.

While serious cardiotoxicity associated with high-dose $\mathrm{CY}$ is rare, it can occur easily under certain clinical conditions, such as excess doses of CY, previous anthracycline treatment, and presence of left ventricular (LV) dysfunction (ejection fraction less than 50\%). It has been reported that a young patient with mediastinal seminoma and a history of radiation therapy caused cardiomyopathy after administration of high-dose CY (5). Radiation therapy to the chest wall may cause direct cardiac toxicity or augment the effects of che- motherapy. Some reports indicated that prior radiation therapy to the mediastinum or chest wall is an independent predictor of cardiotoxicity in patients with lymphoma and breast cancer undergoing high-dose chemotherapy $(6,7)$.

See also p 120.

From the clinical perspective, cardiac monitoring including ECG and ultrasound cardiography (UCG) are important for the detection of early clinical signs of cardiotoxicity. ECG monitoring shows reversible reduction of QRS voltage and/or ST abnormalities. However, those findings are not specific for CY-induced cardiotoxicity. On the other hand, UCG shows a transient increase in LV diastolic/systolic diameter without a concomitant decrease in FS\% and EF\% and abnormalities of diastolic function (E/A mitral Doppler ratio). CY-induced myocarditis increases blood levels of cardiac enzymes (e.g. CK, CK-MB, and troponin-I), however, these parameters can not predict the early phase of cardiotoxicity (8). Recently, the noninvasive method of cardiac magnet resonance imaging (MRI) has proved useful for evaluating LV function and contrast enhancement of cardiac MRI may detect myocardial interstitial edema (9). Although there are no specific predictive parameters of cardiotoxicity, cardiac monitoring in high-risk patients undergoing highdose CY therapy may help to prevent serious cardiotoxicity.

With regard to treatment in an animal model, it has been reported that cyclosporin-A, administered with cyclophosphamide, prevents cardiotoxicity (10), suggesting that CYinduced cardiotoxicity is associated with mitochondrial membrane permeability to $\mathrm{Ca}^{2+}$ and that cyclosporin-A may restore mitochondrial permeability and prevent apoptosis of cardiomyocytes (11). However, clinically there are, as yet, no reports of specific and useful therapies for CY-induced cardiotoxicity. Therefore, to prevent cardiotoxicity in highrisk patients, physicians should consider reducing the dose and using cardiac monitoring to detect the early phase of cardiotoxicity.

Ikuo TANIGUCHI, MD

Division of Cardiology, Jikei University School of Medicine, 3-25-8 Nishishimbashi, Minato-ku, Tokyo 105-8461

\section{References}

1) Santos GW, Sensenbrenner LL, Burke PJ, et al. The use of cyclophosphamide for clinical marrow transplantation. Transplant Proc 4: 
559-564, 1972.

2) Gottdiener JS, Applebaum ER, Ferrans VJ, Deisseroth A, Ziegler J. Cardiotoxicity associated with high-dose cyclophosphamide therapy. Arch Intern Med 141: 758-763, 1981.

3) Goldberg MA, Antin JH, Guiman EC, Rappeport JM. Cyclophosphamide cardiotoxicity: An analysis of dosing as a risk factor. Blood 68 : 1114-1118, 1986.

4) Birchall IW, Lalani Z, Venner P, Hugh J. Fatal haemorrhagic myocarditis secondary to cyclophosphamide therapy. Br J Radiol 73 : $1112-1114,2000$

5) Kamezaki K, Fukuda T, Makino S, Harada M. Cyclophosphamideinduced cardiomyopathy in a patient with seminoma and a history of mediastinal irradiation. Intern Med 44: 120-123, 2005.

6) Brockstein BE, Smiley C, Al-Sadir J, Williams SF. Cardiac and pulmonary toxicity in patients undergoing high-dose chemotherapy for lymphoma and breast cancer: prognostic factors. Bone Marrow Transplant 25: $885-894,2000$
7) Nieto Y, Cagnoni PJ, Bearman SI, Shpall EJ, Matthes S, Jones RB. Cardiac toxicity following high-dose cyclophosphamide, cisplatin, and BCNU (STAMP-I) for breast cancer. Biol Blood Marrow Transplant 6: 198-203, 2000.

8) Morandi P, Ruffini PA, Benvenuto GM, et al. Serum cardiac troponin I levels and ECG/Echo monitoring in breast cancer patients undergoing high-dose $\left(7 \mathrm{~g} / \mathrm{m}^{2}\right)$ cyclophosphamide. Bone Marrow Transplant 28 : 277-282, 2001.

9) Wagner A, Schulz-Menger J, Dietz R, Friedrich MG. Long-term follow-up of patients paragraph sign with acute myocarditis by magnetic paragraph sign resonance imaging. MAGMA 16; 17-20, 2003.

10) al-Nasser IA. In vivo prevention of cyclophosphamide-induced $\mathrm{Ca}^{2+} \mathrm{de}-$ pendent damage of rat heart and liver mitochondria by cyclosporin A. Comp Biochem Physiol A Mol Integr Physiol 121: 209-214, 1998.

11) Beranek JT. Apoptosis contributes to cyclophosphamide-induced cardiomyopathy. Bone Marrow Transplant 29: 91, 2002. 